\title{
The role of breakfast in the diets of Irish adults (18-90y)
}

\author{
$\underline{\text { Ioana Rusu }}^{1,2}$, Laura Kehoe ${ }^{1}$, Breige A. McNulty ${ }^{3}$, Anne P. Nugent ${ }^{4}$, Albert Flynn ${ }^{1}$ and \\ Janette Walton ${ }^{2}$ \\ ${ }^{1}$ School of Food and Nutritional Sciences, University College Cork, Ireland, Cork, Ireland, \\ ${ }^{2}$ Dept. Biological Sciences, Cork Institute of Technology, Ireland, Cork, Ireland, \\ ${ }^{3} U C D$ Institute of Food and Health, University College Dublin, Ireland, Dublin, Ireland and \\ ${ }^{4}$ Institute for Global Food Security, Queens University Belfast, Belfast, United Kingdom
}

\section{Abstract}

Breakfast is often referred to as 'the most important meal of the day' and is consumed after the longest postprandial fast (an overnight fast). Breakfast consumption has been positively linked to many health benefits and has been shown in many studies to be associated with a better diet quality. The aim of this study was to characterise breakfast in Irish adults and to investigate the contribution of breakfast to overall daily nutrient intake.

Analyses were based on the National Adult Nutrition Survey (NANS) (2008-2010) (www.iuna.net). A 4 day semi-weighed food record was used to collect food and beverage intake data from a nationally representative sample of 1500 adults in the Republic of Ireland. Nutrient intakes were estimated using WISP $^{\odot}$ based on UK and Irish food composition data. Breakfast was subjectively defined by the participant and reported in the food diary. In line with recommendations on breakfast studies, this study confined analyses to nutrients derived from breakfast foods and not nutritional supplements. Usual intakes of energy and selected nutrients (from breakfast foods only and total diet (including nutritional supplements)) were calculated via the NCI-method using SAS ${ }^{\mathcal{O}}$ Enterprise Guide.

Breakfast was consumed on at least one recording day by $99 \%$ of participants with an uptake of $93 \%$ of potential breakfast occasions. The mean (SD) energy intake at breakfast was $365(162) \mathrm{kcal}$, contributing on average $19 \%$ to overall daily energy intake. Energy intake from breakfast comprised of 56\% carbohydrate, $14 \%$ fat and $29 \%$ protein. The most frequently consumed foods at breakfast included breakfast cereals, white/brown/wholemeal breads and rolls, butters and fat spreads, jams and marmalades, fruits, eggs, yogurts and some meats. The most frequently consumed beverages included teas, coffees, 'fruit juices \& smoothies', waters and milk (in teas/coffees, with cereals and as a beverage). The contribution of breakfast to total daily nutrient intakes was $25 \%$ for carbohydrate, $16 \%$ for fat and $16 \%$ for protein. Breakfast also contributed to total daily intakes of dietary fibre (22\%), total sugars (28\%), saturated fat (18\%), B-vitamins (20-32\%), vitamins C (23\%), D (24\%), E (19\%) calcium $(28 \%)$, iron $(26 \%)$ and sodium $(18 \%)$.

Breakfast was widely consumed among Irish adults and was typically a nutrient dense meal which contributed significantly to total dietary intakes of a number of important macro- and micro- nutrients but also contributed to total sugars and relatively small proportions of total fat, saturated fat, and sodium.

\section{Conflict of Interest}

There is no conflict of interest. 